Z. klin. Chem. u. klin. Biochem.

9. Jg., S. 155-163, März 1971

\title{
Tryptophan-Stoffwechseluntersuchungen bei Pyridoxin-abhängigen Krämpfen
}

\author{
Von W. KOCHEN \\ Aus dem Isotopenlabor der Universitäts-Kinderklinik (Direktor: Prof. Dr. H. Bickel) Heidelberg
}

(Eingegangen am 16. November 1970)

Aus einer Familie mit 5 Kindern verstarben zwei im Säuglingsalter an Therapie-resistenten Krämpfen, zwei Kinder sind klinisch gesund, ein Kind leidet an Vitamin-B ${ }_{6}$-abhängigen Krämpfen. Bei den drei lebenden Kindern (2., 4. und 5. Kind) werden die Abbauprodukte des Tryptophan im Urin sowohl vor wie nach Belastung mit L-Tryptophan bestimmt. -

Das an Vitamin- $\mathrm{B}_{6}$-abhängigen Ktämpfen leidende vierte Kind zeigt unter Vitamin- $\mathrm{B}_{8}$-Langzeit-Therapie ein im wcsentlichen normales Ausscheidungsmuster der Tryptophan-Metabolite. Nach Absetzen der Vitamin- $\mathrm{B}_{6}$-Therapie nimmt dic Ausscheidung der oxydativen Abbauprodukte ab. Zwölf Tage nach Unterbrechung der Therapie setzt ein Grand-Mal Anfall ein, der nach Injektion von $200 \mathrm{mg}$ Pyridoxin innerhalb von Minuten sistiert. Das Ausscheidungsmuster der Metabolite bleibt aber weiterhin anormal, eine Normalisierung tritt erst zwischen dem 10. und 20. Tag nach Wiederaufnahme der Therapie ein. In dem Urin vor dem Krampfanfall wird eine Substanz vermehrt ausgeschieden, die als 8-Hydroxy-chinaldinsäure identifiziert wird. Unter Vitamin- $\mathrm{B}_{6}$-Therapie kann dieser Metabolit nicht nachgewiesen werden. Es wird ein Zusammenhang zwischen der Bildung dieses Metaboliten und dem geschilderten Krampfgeschehen vermutet. Nach bisherigen Untersuchungen wird die 8-Hydroxy-chinaldinsäure im Urin von gesunden Kindern und Erwachsenen nicht gefunden.

\section{An investigation of tryptophan metabolism in a case of convulsions caused by pyridoxine dependency}

In a family with 5 children, two children died in infancy of convulsions which were resistant to therapy, two children are clinically healthy and one child suffers from convulsions which are dependent on vitamin $\mathrm{B}_{6}$. The amounts of tryptophan metabolites in the urine of the latter three children ( 2 nd, 4 th and 5 th child) were determined before and after loading with L-tryptophan. -

The 4 th child which suffers from vitamin- $\mathrm{B}_{6}$-dependent convulsions shows an essentially normal elimination pattern of the tryptophan metabolites under vitamin- $B_{6}$-therapy. When the vitamin- $B_{6}$-therapy is stopped, however, the amounts of oxidative degradation products of tryptophan are markedly decreased. Twelve days later a Grand-Mal seizure sets in, which ceases immediately when $200 \mathrm{mg}$ pyridoxine are injected. The elimination pattern remains abnormal. A normal excretion is observed between the 10 th and 20 th day after vitamin- $\mathrm{B}_{6}$-therapy is resumed. A substance which is identified as 8 -hydroxy-quinaldic acid is found in increased amounts in the urine before the seizures. This substance cannot be detected in the urine during vitamin- $\mathrm{B}_{6}$-therapy. It has not been found as yet in the urine of healthy children or adults. It is suggested that a connection exists between the seizure and the appearance of 8-hydroxy-quinaldic acid in the urine.

Bei den „Pyridoxin-abhängigen Krämpfen“ im Säuglingsalter handelt es sich um ein metabolisch-genetisches Anfallsleiden. Diese Krämpfe können nur durch eine fortgesetzte, mehr oder weniger große Zufuhr von Vitamin $B_{6}$ kupiert werden. Unter einer frühzeitig eingesetzten Vitamin- $B_{6}$-Dauertherapie verläuft die weitere Entwicklung des Säuglings normal. Fehldiagnose oder zu spät gestellte Diagnose führen zum Tod oder zu schwersten hirnorganischen Defekten.

Die Pathogenese ist ungeklärt. Allgemeine labordiagnostische Untersuchungen ergaben bisher keine gezielten Hinweise. Die mehrfach geäußerte Annahme einer „Vitamin- $B_{6}$-Verwertungsstörung“ (1) kann wissenschaftlich nicht - oder noch nicht - belegt werden. Die Serumkonzentration von Pyridoxal-5-phosphat liegt im Normbereich $(2,3)$, die Ausscheidung der 4-Pyridoxinsäure ist dagegen vermindert (3).

Bisher sind in der gesamten Literatur 19 Fälle von Pyridoxin-abhängigen Krämpfen beschrieben worden (1). Diesen Vitamin- $B_{6}$-abhängigen Krämpfen (Vitamin- $\mathrm{B}_{6}$-dependency) stehen die Vitamin- $\mathrm{B}_{6}$-Mangelkrämpfe (Vitamin- $B_{6}$-deficiency) gegenüber. Zur Differentialdiagnose hat sich seit Jahren der sog. Xanthurensäure-Test nach Tryptophanbelastung eingebürgert. Im Gegensatz zur Vitamin- $B_{6}$-Abhängigkeit wird bei Vitamin- B $_{6}$-Mangel die Xanthurensäure anormal vermehrt ausgeschieden. Jedoch sind gegen diesen Test erheblich Bedenken zu erheben (4).

Die Annahme einer Vitamin- $\mathrm{B}_{6}$-Verwertungsstörung könnte bei denjenigen enzymatischen Reaktionen geprüft werden, die Pyridoxal-5-phosphat als Cofaktor benötigen. Als ein geeignetes System bietet sich der Tryptophan-Abbau an, bei dem die enzymatischen Spaltungen von Kynurenin bzw. 3-Hydroxy-kynurenin zu Anthranilsäure bzw. 3-Hydroxy-anthranilsäure, die Bildung von Kynurensäure und Xanthurensäure, die Decarboxylierungen von Chinolinsäure zu Nicotinsäure und von 5-Hydroxy-tryptophan zu Serotonin, Pyridoxal-5-phosphat-abhängig sind.

Im folgenden werden die Ausscheidungsprodukte des Tryptophans im Urin bei einem Kind mit eindeutig nachgewiesenen Vitamin- $B_{6}$-abhängigen Krämpfen untersucht. Analoge Stoffwechselstudien werden bei zwei klinisch gesunden Geschwisterkindern durchgeführt.

\section{Kasuistik}

Unter den noch lebenden Kindern der Familie befindet sich ein Kind, bei dem in unserer Klinik ein Pyridoxin-abhängiges Anfallsleiden nachgewiesen wurdc (4). Bei zwei im frühen Säug- 
lingsalter an therapieresistenten Krämpfen verstorbenen Gcschwistern ist die genaue Diagnose nicht bekannt, doch licgt dic Vermutung nahe, daß bei ihnen cine analoge Vitamin- $\mathrm{B}_{6}-\mathrm{Ab}$ hängigkeit bestanden hat.

Die Eltern sind klinisch gesund, erbliche Krankheiten sind in ihrer Aszendenz nicht festzustellen. Ihr erstes Kind begann am 9. Lebenstag an Krämpfen zu leiden, die therapieresistent waren. Der Tod trat nach 10 Wochen ein. Das sechs Jahre später geborene dritte Kind zeigte ebenfalls seit dem 9. Lebenstag Krämpfe. Eine geringe Besserung wurde nach Verabreichung von Comital ${ }^{1}$ ) beobachtet. Im EEG fand sich eine Hypsarrhythmie. Im Verlauf der ersten Monate wurde ein schwerer Cerebralschaden mit Tetraspastik festgestellt, eine Besserung durch ACTH trat nicht ein. Nach 9 Monaten verstarb das Kind im status epilepticus. Die noch lebenden Geschwister (2., 4. und 5. Kind) wurden in der hiesigen Klinik untersucht. Das zweite Kind zeigte bisher eine unauffällige Entwicklung. Seit der Einschulung wurden von den Eltern Schlafstörungen beobachtet. Der IQ beträgt nach Kramer 0,91 , die Merkfähigkeit und die visomotorischen Leistungen sind um 1-2 Jahre rückläufig. Klinisch und neurologisch besteht kein krankhafter Befund. Das EEG zeigt eine geringe allgemeine Verlangsamung, es ist jedoch nicht eindeutig pathologisch.

Das vierte Kind zeigte zuerst eine normale psychomotorische Entwicklung, bis im 3. Lebensmonat plötzlich Schreiattacken auftraten, denen nach 3 Tagen Grand-Mal Anfälle von 2 Stdn. Dauer folgten. Trotz Verabreichung von Luminaletten ${ }^{1}$ ) wiederholten sich die Anfälle in den folgenden Tagen. Bei Aufnahme in die hiesige Klinik konnte ein bisher therapieresistenter Anfall mit $375 \mathrm{mg}$ Benadon $^{1}$ ) i. v. durchbrochen werden. In der Folgezeit wurde unter täglich $40 \mathrm{mg}$ Pyridoxin ein normales EEG erhalten. Sechs Tage nach Vitamin- $B_{6}$-Entzug wurde wieder ein pathologisches EEG registriert. Unter täglicher Vitamin- $\mathrm{B}_{6}-$ Zufuhr rerlief nunmehr die psychmotorische Entwicklung normal, Krampfanfälle wurden nicht mehr beobachtet.

Das fünfte Kind zeigte klinisch und neurologisch keine krankhaften Befunde. Alle EEG Ableitungen waren normal.

\section{Methodik}

Der Sammelurin wird tiefgefroren bis zur Aufarbeitung aufbewahrt. Die Auftrennung der Tryptophan-Metabolite erfolgt säulenchromatographisch auf dem starksauren Kationenaustauscher Amberlite IR-120/AS. Eluiert wird mit zwei flüchtigen wäßr. Ameisensäure/Pyridin Gemischen vom pH 2,20 und 2,60. Die gefriergetrockneten Fraktionen werden dünnschichtchromatographisch untersucht (Cellulose Machery u. Nagel mit Fluoreszenzindikator, Laufmittel Butanol/Eisessig/Wasser 4:1:5 $\nabla / v)$. Die quantitative Auswertung nach einer 2. Chromatographie erfolgt direkt auf der Dünnschichtplatte (Cellulose und Polyamid Fertigplatten von Merck) mit Hilfe des Dünnschicht-Fluorometers nach Turner (Firma Camag, Schweiz) und/oder nach Anfärbung und Auswertung mit dem Dünnschicht-Spektralphotometer der Firma Zeiss. Einzelheiten über den Trennungsgang der oxydativen Metabolite siehe Koches und Mitarbeiter (5), über die Trennung der Indolverbindungen siehe KocheN (6). In Zweifelsfällen wird zur sicheren Identifizierung der Indolverbindungen und zur Unterscheidung von Anthranilsäure und 3-Hydroxyanthranilsäure die Gaschromatographie angewendet (6).

\section{Ergebnisse}

Untersuchungen bei dem Kind mit Vitamin$\mathrm{B}_{6}$-abhängigen Krämpfen

Drei Jahre nach Ausbruch der Vitamin- $\mathrm{B}_{6}$-abhängigen Krämpfe werden bei dem 4. Kind die TryptophanStoffwechseluntersuchungen durchgeführt. Vom 3. Le-

1) Comital = Diphenylhydantoin + N-Methyl-äthylphenyl-barbitursäure, Luminaletten = Phenyläthyl-barbitursäure, Benadon = Pyridoxinhydrochlorid. bensmonat an hatte das normal entwickelte Kind täglich $40 \mathrm{mg}$ Pyridoxin oral erhalten. Unter Fortführung dieser Therapie wird der Urin (Basalurin) untersucht und die L-Tryptophan Belastung $(0,10 \mathrm{~g} / \mathrm{kg}$ KG) durchgeführt. Der Basalurin (Abb. 1, A) zeigt eine im Normbereich liegende Ausscheidung der Metabolite des oxydativen Abbaus, wobei zu bemerken ist, daß alle Hauptmetabolite mit Ausnahme von Anthranilsäưre nachgewiesen werden können. Nur Kynurenin muß als erniedrigt angesehen werden. Nach unseren bisher vorliegenden Untersuchungen werden bei Kindern und auch bei Erwachsenen (5) nur in seltenen Fällen alle diese 6 Metabolite des oxydativen Abbaus gleichzeitig im Urin ausgeschieden. Tabelle 1 gibt die Normalwerte wieder bei Kindern $(n=10)$ im Alter zwischen $1 / 4$ und 7 Jahren sowohl vor wie nach Belastung mit L-Tryptophan.

Tab. 1

Normalwerte der Tryptophanmetabolite im Harn von Kindern vor und nach Tryptophanbelastung ( $100 \mathrm{mg} / \mathrm{kg} \mathrm{KG)}$

\begin{tabular}{lcc}
\hline & $\begin{array}{c}\text { Vor Belastung } \\
\text { mg/24-Stdn.-Urin }\end{array}$ & $\begin{array}{c}\text { 24 Stdn. nach } \\
\text { Belastung } \\
\text { Normalwert ohne } \\
\text { Belastung mal }\end{array}$ \\
\hline $\begin{array}{l}\text { Kynurenin } \\
\text { Kynurensäure }\end{array}$ & $0,6-2,6$ & $10-20$ \\
3-Hydroxy-kynurenin & $1,0-2,5$ & $3-5$ \\
Xanthurensäure & $0,2-0,7$ & $3-4$ \\
Anthranilsäure & $0,5-2,0$ & $<5 \mathrm{mg}$ \\
3-Hydroxy-anthranilsäure & $<1,0$ & 8 \\
\hline
\end{tabular}

Die quantitative Ausscheidung der Indolverbindungen liegt im Normbereich mit Ausnahme der 5-Hydroxyindolessigsäure, deren Konzentration bei Kontrollkindern $(n=5)$ immer niedriger ist als die der Indolessigsäure.

Nach Tryptophan-Belastung (Abb. 1,B) liegt die vermehrte Ausscheidung des Kynurenin und der Kynurensäure im Normbereich, die der 3-Hydroxy-anthranilsäure ist erhöht. Auffallend ist die nur geringe Erhöhung des 3-Hydroxy-kynurenin. Unter den Indolen wird als neuer Metabolit die Indolmilchsäure ausgeschieden. Bemerkenswert ist die unerklärliche Abnahme der 5-Hydroxy-indolessigsäure. Aus Abbildung 1 ersieht man ferner, daß die Metabolite das Maximum ihrer Ausscheidung innerhalb der ersten $9 \mathrm{Stdn}$. nach Belastung haben, während in der restlichen 24 Stdn.Periode nur noch sehr geringe Mengen von Xanthurensäure, Indolessigsäure, 5-Hydroxy-indolessigsäure und Tryptophan ausgeschieden werden.

Nunmehr wird die tägliche Vitamin- $\mathrm{B}_{6}$-Therapie unterbrochen. Die Urinuntersuchung nach 6 Tagen zeigt ein signifikantes Absinken der Metaboliten=Ausscheidung, das bis zum völligen Versiegen von 3-Hydroxy-kynurenin, Kynurensäure und von 3-Hydroxy-anthranilsäure führt. Selbst die 5-Hydroxy-indolessigsäure, die unter hoher Vitamin- $\mathrm{B}_{6}-$ Zufuhr massiv ausgeschieden wird, sinkt nunmehr auf Null. Dagegen steigt die Tryptophan-Ausscheidung auf einen Wert wie nach einer Belastung an (Abb. 1, C).

Dieses Ausscheidungsmuster kann nicht als normal angesehen werden. Bei gesunden Kindern dieser 


\section{Gastrokamera-Untersuchung}

\section{Grundlagen}

Untersuchungstechnik, Bildbeurteilung, Ergebnisse ·

mit den Referaten des Ersten Gastrokamera-Seminars, 13._15. Juni 1969, Berlin

\section{Herausgegeben von Dozent Dr. H. Oshima}

Gastprofessor an der Freien Universität Berlin, Nippon-Ika-Universität Tokyo, Präsident der European Association for Gastrokamera Diagnosis, unter Mitarbeit von Dr. W. BergemanN, Berlin

Oktav. VIII, 146 Seiten. Mit 33 Abbildungen. 1971. Kartoniert DM 20,-

Die Gastrokamera-Untersuchung, ein schnelles, einfaches und beschwerdearmes Verfahren zur intragastralen farbigen Fotografie für die Magen-Diagnostik hat in Europa in den letzten Jahren eine zunehmende Bedeutung erlangt.

Vom 13.-15. 6. 1969 wurde das Erste Gastrokamera-Seminar im Klinikum Steglitz der Freien Universität Berlin veranstaltet.

Die verschiedenen Gastrokamera-Modelle, die Untersuchungstechnik und besonders die Beurteilungsmethode sowie -kriterien wurden als Schwerpunkte behandelt.

Durch die Berichte der verschiedenen Untersucher aus 6 europäischen Ländern wurde der derzeitige Stand der Gastrokamera-Diagnostik in Europa dargelegt.

Die etwa 300 Teilnehmer aus 9 europäischen Ländern bewiesen das rege Interesse an dieser Untersuchungsart.

An einer weiter zunehmenden Bedeutung dieses diagnostischen Verfahrens ist bei der Häufigkeit von Magenerkrankungen, insbesondere des Magenkrebses nicht zu zweifeln. Dazu soll die Herausgabe dieser Verhandlungsberichte als Leitfaden der Gastrokamera-Untersuchung beitragen.

In Vọbereteitung:

\section{Europäisches Gastrokamera-Symposion}

vom 27. 2.-1.3. 1970

von H. Oshima und W. BergemanN

Etwa 176 Seiten. Etwa DM 24,-

\section{Walter de Gruyter • Berlin · New York}




\section{BIOCHIMIE}

Edité par la Société de Chimie Biologique

tel est le titre

sous lequel paraîtra à partir de 1971

le „BULLLETIN DE LA SOCIÉTÉ DE CHIMIE BIOLOGIQUE“

\section{SECRÉTARIAT}

de la Société de Chimie Biologique

J. P. EBEL, Secrétaire Général (Relations Extérieures)

R. PERLES, Secrétaire Général

\section{REDACTION}

F. GROS, Secrétaire scientifique

F. PERCHERON, Secrétaire à la Publication

J. NUNEZ, Secrétaire à l'Information

Y. RAOUL, Secrétaire à l'Edition

SECRETARIAT et REDACTION: 4 Avenue de l'Observatoire, PARIS $6^{\circ}$

12 FASCICULES

ABONN̦EMENTS: FRANCE et ZONE FRANC: $150 \mathrm{ffrcs}$ - BELGIQUE: 1.687,- frcs - AUTRES PAYS: 186,一 ffrcs

MASSON et Cie, Editeurs · 120 Boulevard St Germain · PARIS 6ème

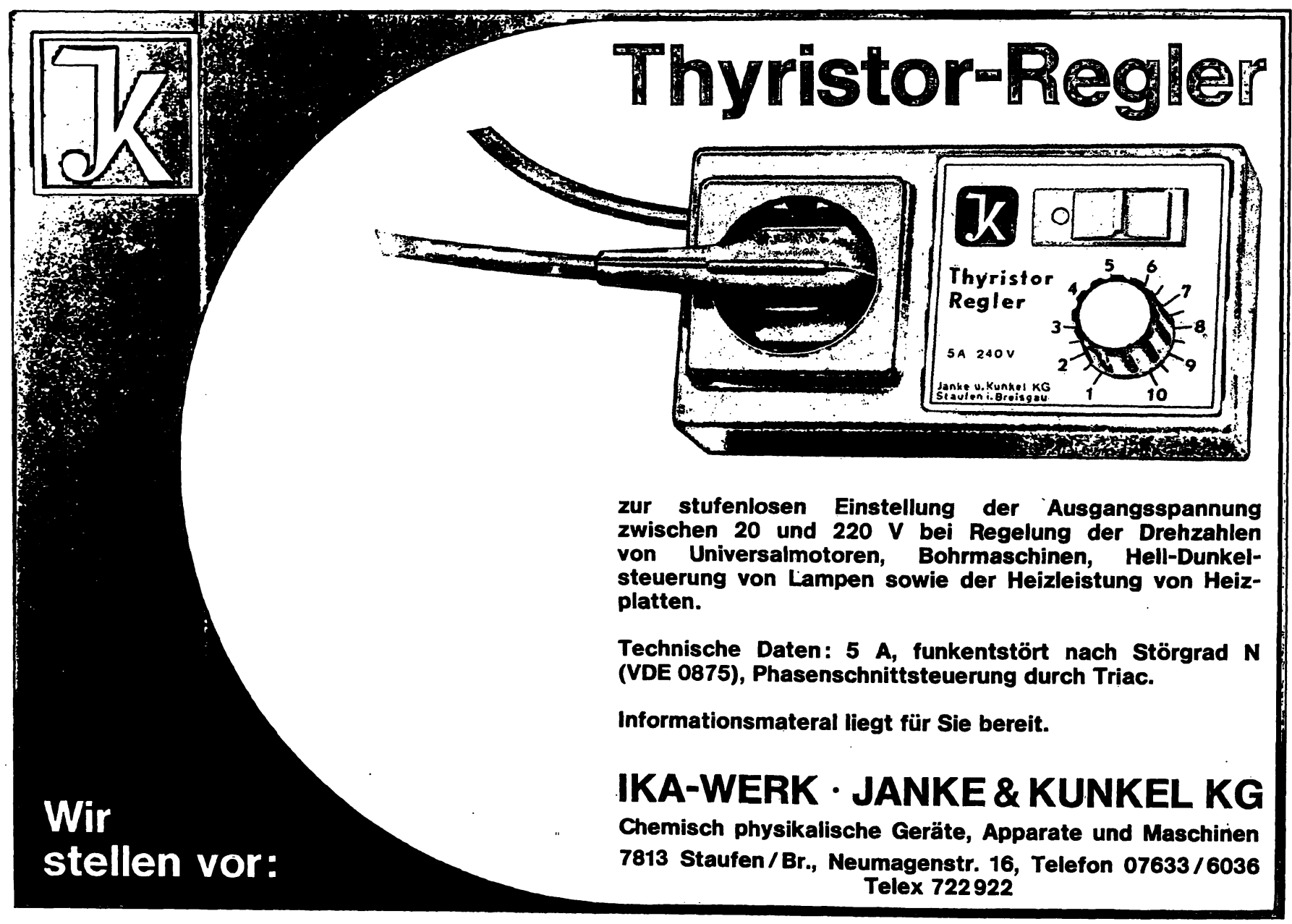


Abb. 1

Viertes Kind, Vitamin-B,-abhängige Krämpfe: Ausscheidung von Tryptophan-Metaboliten im Urin

A = Basalurin bei Gabe von $<40 \mathrm{mg}$ Pyridoxin/Tag

$\mathrm{B}=$ Belastung mit 1,5 g L-Tryptophan, Ausscheidung in den ersten 9 Stdn.; erfolgt in der restlichen 24-Stdn.-Periode noch eine weitere Ausscheidung, ist diese Angabe schraffiert

$\mathrm{C}=$ Basalurin, 6 Tage nach Absetzen von Pyridoxin

$D=$ Basalurin, 12 Tage nach Absetzen von Pyridoxin (Grand-Mal Anfall, $200 \mathrm{mg}$ Pyridoxin iv.)

$\mathrm{E}=$ Basalurin, 9 Tage nach Krampfanfall, wieder Pyridoxin Therapie. Annahme unter $B$

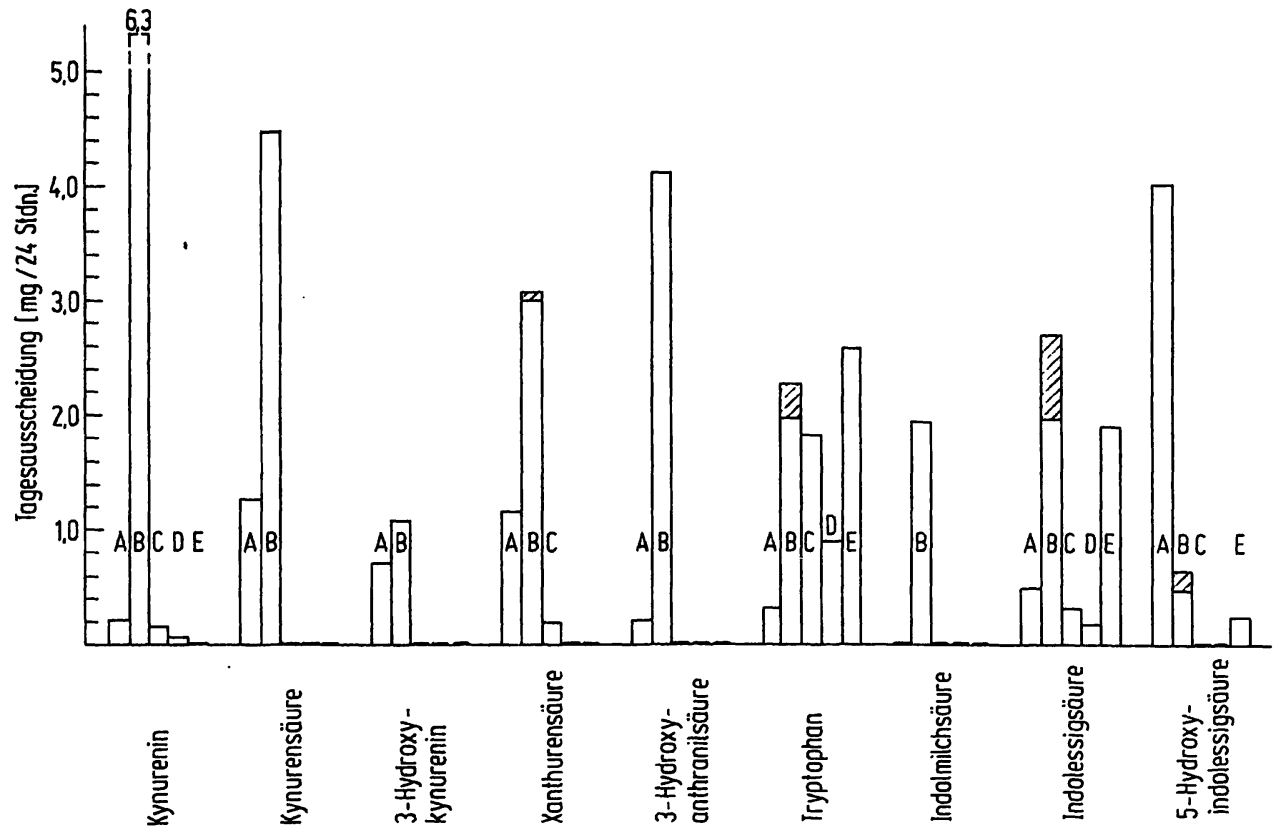

Altersstufe finden wir gelegentlich auch nur Kynurenin und Xanthurensäure, die Werte liegen dann bei etwa $1 \mathrm{mg} / 24 \mathrm{Stdn}$. und eine Tryptophan-Ausscheidung über $1 \mathrm{mg}$ ist nicht $\mathrm{zu}$ beobachten.

$\mathrm{Zu}$ diesem Zeitpunkt (6 Tage nach Absetzen von Vitamin $B_{6}$ ) können im EEG keine Abnormalitäten festgestellt werden. Der Einfluß der Pyridoxin-Therapie auf den Tryptophan-Stoffwechsel ist also bei diesem Kind mit Vitamin- $\mathrm{B}_{6}$-abhängigen Krämpfen signifikant sichtbar.

Zwölf Tage nach Absetzen der Vitamin- $B_{6}-T_{\text {Therapie }}$ tritt eine Krampfanfall auf, der durch $200 \mathrm{mg}$ Pyridoxin iv. nach wenigen Minuten kupiert werden kann. Die Untersuchung des nach dem Grand-Mal Anfall folgenden 24 Stdn.-Urins zeigt trotz der hohen Vitamin$\mathrm{B}_{6}$-Injektion keine Erhöhung der Metaboliten-Ausscheidung.

Xanthurensäure kann nicht mehr nachgewiesen werden, die Ausscheidung des Kynurenin und der Indolessigsäure sinken weiter ab (Abb. 1, D). Das Kind hatte ab 3. Tag nach dem Anfall - es hatte sich inzwischen klinisch wieder etholt - $5 \mathrm{mg}$ Vitamin-B $6 /$ Tag erhalten. Jedoch mußte die Dosis auf $10 \mathrm{mg} / \mathrm{Tag}$ erhöht werden, da nur unter dieser Dosierung die im EEG registrierbaren geringfügigen Abnormalitäten wieder verschwanden.

Am 9. Tag nach dem Anfall, d. h. 6 Tage nach Wiederaufnahme der Vitamin- $\mathrm{B}_{6}$-Therapie, wird immer noch kein normales Ausscheidungsmuster festgestellt. Metabolite des oxydativen Abbauweges können dünnschichtchromatographisch überhaupt nicht nachgewiesen werden. Dagegen werden Tryptophan und Indolessigsäure vermehrt ausgeschieden (Abb. 1, E). Eine Urinuntersuchung 3 Wochen nach dem Anfall ergab erst wieder ein Ausscheidungsmuster, das als normal $\mathrm{zu}$ bezeichnen ist und in Übereinstimmung steht mit den Ergebnissen von Abb. $1_{3} \mathrm{~A}$.

\section{Untersuchung unbekannter Stoffivechselprodukte}

Durch die angewandte analytische Methode, unter konstanten Bedingungen der Säulenchromatographie die Fraktionen dünnschichtchromatographisch zu untersuchen, ist ein Vergleich der Urine möglich, ohne $\mathrm{da}$ die Substanzzonen im einzelnen identifiziert werden können. Diese Methode zeigt ihren Wert besonders bei der Urinuntersuchung vor und nach Tryptophan-Belastung. Außer den uns z. Z. bekannten Metaboliten des Tryptophan werden bei dem Kind mit Vitamin- $\mathrm{B}_{6}$-abhängigen Krämpfen nach Tryptophan-Belastung 17 neue Metabolite beobachtet, die vor der Belastung dünnschichtchromatographisch nicht nachgewiesen werden können. Diese Substanzen fluoreszieren alle auf der Dünnschichtplatte. Aufgrund der UV-Spektren und der Anfärbergebnisse wird bei 12 Metaboliten ein Zusammenhang zum TryptophanStoffwechsel angenommen.

Zwei Metabolite mögen näher beschrieben werden, von denen einer identifiziert werden konnte.

In dem Urin unter täglicher Vitamin-B ${ }_{6}-$ Zufuhr (vgl. Abb. 1, A) wird eine sehr stark fluoreszierende Substanz dünnschichtchromatographisch nachgewiesen. Sie wird von der Amberlite Kationenaustauschersäule mit dem Puffersystem Ameisensäure/Pyridin/Wasser, pH 2,60, sofort eluiert. Die Isolierung der reinen Substanz gelang bisher nicht. Der Schmelzpunkt liegt bei $180^{\circ}$ unter Zersetzung und Gasentwicklung. Das Maximum der Fluoreszenz liegt bei $435 \mathrm{~nm}$ bei einer Anregung mit $365 \mathrm{~nm}$. Die Substanz wird mit Dibromchinonchlorimid blau und mit diazotierter Sulfanilsäure rot angefärbt. Die Ausscheidung dieses Metaboliten nimmt nach Tryptophan-Belastung (unter. Vitamin- $\mathrm{B}_{6}$-Therapie) stark $a b$. Sechs Tage nach Vitamin- $B_{6}-$ Entzug kann er noch in geringen Mengen nachgewiesen werden. Nach Wiederaufnahme der Vitamin- $\mathrm{B}_{6}$-Behandlung tritt zuerst keine vermehrte Ausscheidung ein. $\mathrm{Zu}$ dem Zeit- 
punkt, in dem der Tryptophan-Stoffwechsel wieder normalisiert ist (etwa 3 Wochen nach Wiederaufnahme der Vitamin- $\mathrm{B}_{6}$-Therapie) erfolgt wieder eine massive Ausscheidung.

Bei den Urinuntersuchungen des Kindes mit Vitamin$\mathrm{B}_{6}$-Abhängigkeit fiel auf der Dünnschichtplatte eine Zone auf, die nach Absetzen des zusätzlichen Vitamin$B_{6}$ zuerst nur sehr schwach zu beobachten war, deten Menge aber in dem Urin einen Tag vor dem Krampfanfall stark vermehrt $z u$ finden war. Dies war der Grund, die Substanz zu isolieren. Sie konnte eindeutig als 8-Hydroxy-chinaldinsäure identifiziert werden.<smiles></smiles>

Isolierung: $70 \mathrm{~m} l$ gefriergetrockneter Urin werden auf einer Amberlite-Kationenaustauschersäule (innerer $\varnothing 1 \mathrm{~cm}$, Füllhöhe $30 \mathrm{~cm}$ ) chromatographiert. Mit $100 \mathrm{~m} l$ eines $0,3 \mathrm{M}$ wäßr. Pyridin/ Ameisensäure-Puffers ( $\mathrm{pH}$ 2,60) werden mehrere Pyridin- und Chinolinderivate aus dem Tryptophan-Stoffwechsel eluiert, mit einem analogen Puffer vom $\mathrm{pH}$ 5,60 erscheinen dann Anthranilsäure, 3-Hydroxy-anthranilsäure, Kynurenin und 3-Hydroxykynurenin. Zwischen den beiden letzten Metaboliten wird die 8-Hydroxy-chinaldinsäure eluiert. Die Elution erfolgt in den Fraktionen mit $70-90 \mathrm{~m} /$ Durchfluß des 2. Puffersystems. Insgesamt wurden aus $1,5 l$ Urin $7 \mathrm{mg}$ reine Substanz gewonnen. Die weitere Reinigung erfolgt dünnschichtchromatographisch.

Tab. 2

Dünnschichtchromatographische Reinigung von 8-Hydroxy-chinal-

\begin{tabular}{ccccc}
\hline & $\begin{array}{c}\text { Butanol/Eisessig/ } \\
\text { Wasser 4:1:5(v/v) }\end{array}$ & $\begin{array}{c}\text { Dimethylformamid/ } \\
\text { Wasser } 7: 3(\mathrm{v} / \mathrm{v})\end{array}$ \\
\hline $\begin{array}{c}\text { Trägermaterial } \\
R_{\mathrm{F}} \text {-Wert }\end{array}$ & $\begin{array}{c}\text { Cellulose } \\
0,75\end{array}$ & $\begin{array}{c}\text { Polyamid } \\
0,50\end{array}$ & $\begin{array}{c}\text { Cellulose } \\
0,75\end{array}$ & $\begin{array}{c}\text { Polyamid } \\
0,07\end{array}$ \\
\hline
\end{tabular}

Der Metabolit fluoresziert schwach rosa und wird mit diazotierter Sulfanilsäure orange angefärbt.

Die ersten Hinweise über die Struktur werden durch das UV-Absorptionsspektrum erhalten (Abb. 2).

Das Spektrum zeigt in äthanol. Lösung (neutrales Medium) 3 Absorptionsbanden im Bereich zwischen $355 \mathrm{~nm}$ und $255 \mathrm{~nm}$.
Die intensivste Bande liegt bei $255 \mathrm{~nm}$. Die langwelligeren Banden bei 355 und $310 \mathrm{~nm}$ haben eine wesentlich geringere Extinktion. Solche UV-Spektren sind charakteristisch für Hydroxy-chinoline und Hydroxy-iso-chinoline, ferner für Naphthole (7). Wird die Messung in $0,01 \mathrm{~N}$ äthanol. $\mathrm{NaOH}$ wiederholt, so tritt eine bathochrome Verschiebung ein, gleichzeitig exhöhen sich die Extinktionen. Die beiden längerwelligen Banden werden von 355 auf $395 \mathrm{~nm}^{\prime}$ und von 310.auf $343 \mathrm{~nm}$ verschoben, die kurzwelligste Bande von 255 auf $267 \mathrm{~nm}$. Die bathochrome Verschiebung wäre durch die Uberführung eines 'Naphthols in das Anion zu erklären. In saurem Milieu $(0,01 \mathrm{~N}$ äthanol. $\mathrm{HCl})$ ist dagegen eine Bandenverschiebung nicht festzustellen.

Diese beiden Beobachtungen stehen auch dann mit einer Chinolin- bzw. Iso-chinolinstruktur in Übereinstimmung, wenn eine Protonierung des N-Atoms aus sterischen Gründen oder durch Substituenten-Effekte verhindert wird. Diese Interpretation ist von besonderem Interesse, da sich aus dem Massenspektrum eine Chinolinstruktur ableiten läßt. Zur weiteren $\mathrm{Ab}$ klärung kann der Befund von BIRRINGer (8) angeführt werden, daß bei dem 3- bzw. 4-Methylester der 8Hydroxy-chinaldinsäure in saurem Milieu eine Bandenverschiebung eintritt, nicht dagegen bei dem 2-Methylester. Bei einer 2-Stellung der Carboxylgruppe ist eine sterische Abschirmung des $\mathrm{N}$-Atoms anzunehmen.

Für die Aufnahme der folgenden Spektren wird die Substanz nochmals über einer Kieselgelsäule gereinigt, anschließend erfolgte Sublimation bei $150^{\circ}$ und $10^{-3}$ Torr. Das IR-Spektrum gibt weitere Hinweise (Abb. 3).

Das Spektrum zeigt ein kondensiertes aromatisches System an. Die aromatischen Gerüstschwingungen $\nu_{\mathrm{C}}=\mathrm{C}$ sind den Banden bei $1616,1595,1500$ und $1460 \mathrm{~cm}^{-1}$ zuzuordnen (9). Die aromatischen Valenzschwingungen $\nu_{\mathrm{C}-\mathrm{H}}$ dex Protonen exscheinen schwach bei $3065 \mathrm{~cm}^{-1}$, die Deformationsschwingungen $\nu_{\mathrm{C}-\mathrm{H}}$ (,,in plane“) bei 1137, 1088 und $998 \mathrm{~cm}^{-1}$, die entsprechenden „out of plane“ Schwingungen unterhalb von $900 \mathrm{~cm}^{-1}$. Die Anwesenheit einex Hydroxylgruppe wird durch die $\nu_{\mathrm{O}-\mathrm{H}}$ Bande bei $3270 \mathrm{~cm}^{-1}$ angezeigt. (Die Rechtsverschiebung und die Schärfe dieser Bande dürfte auf die Protonenaffinität des benachbarten $\mathrm{N}$-Atoms zurückzuführen sein.) Die Zuordnung der $\nu_{\mathrm{C}-\mathrm{OH}}$ Schwingung im Bereich zwischen 1200 und $1300 \mathrm{~cm}^{-1}$ ist nicht eindeutig zu treffen. Eine intensive Bande bei $1645 \mathrm{~cm}^{-1}$ weist auf eine Carboxylgruppe hin $\left(\nu_{\mathrm{C}=0}\right), \nu_{\mathrm{C}-\mathrm{O}}$ asym. liegt bei $1295, \nu_{\mathrm{C}-0}$ sym. bei $1150 \mathrm{~cm}^{-1}$. Die OH-Schwingung der Carboxylgruppe liegt bei etwa $2460 \mathrm{~cm}^{-1}$. Bezüglich einer Aussage über den Substitutionstyp des aromatischen Systems sind nur Hinweise möglich.

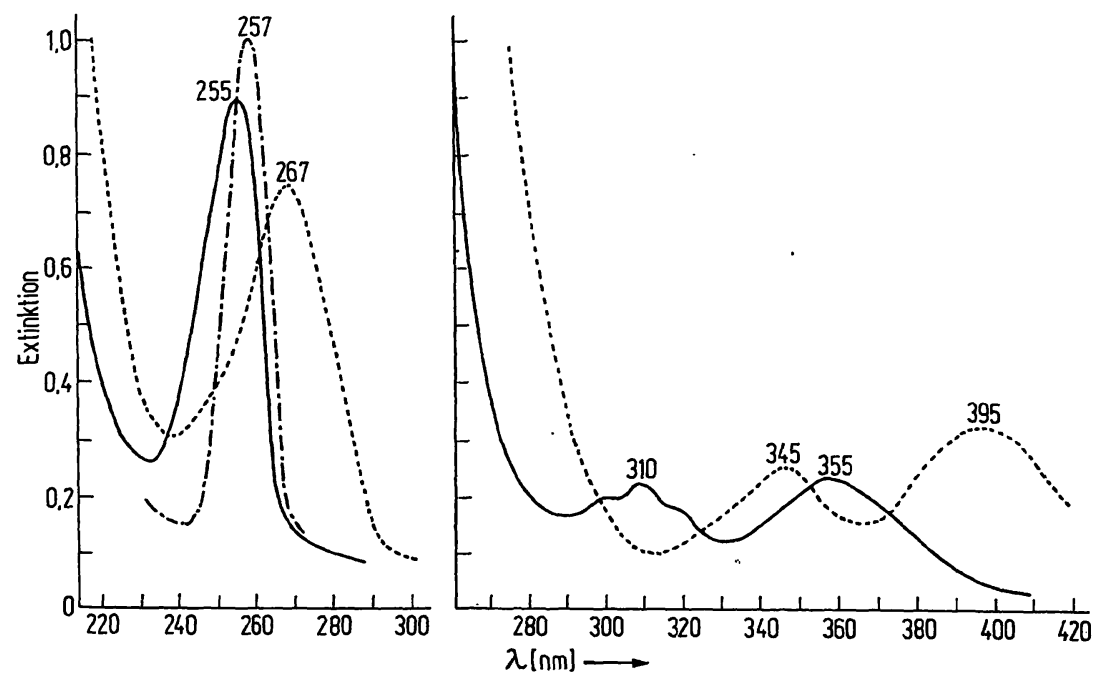

Abb. 2

UV-Absorptionsspektrum der isolierten 8-Hydroxy-chinaldinsäure in Äthanol $(\longrightarrow, 0,01 \mathrm{~N}$ äthanol. $\mathrm{HCl}(-\ldots-\infty)$ und in $0,01 \mathrm{~N}$ äthanol. $\mathrm{NaOH}$ $(\cdots \cdot)$, aufgenommen mit Spektrophotometer Cary 15 
Abb. 3

IR-Absorptionsspektrum der isolierten 8-Hydroxychinaldinsäure, Aufnahme: $0,74 \mathrm{mg}$ in $150 \mathrm{mg} \mathrm{KJ}$ mit dem PE-Spektralphotometer 621

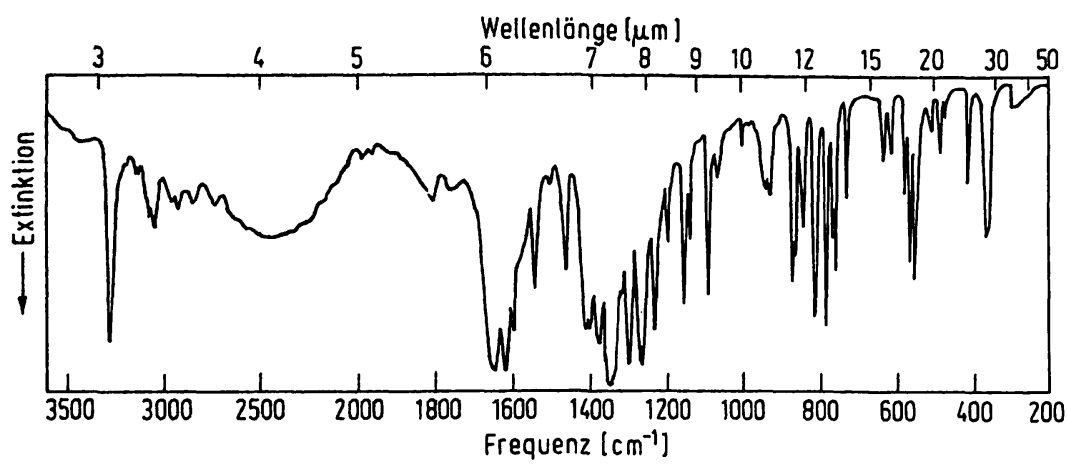

Weitere Aufklärung bringt das Massenspektrum (Abb. 4). Der Molekularpeak zeigt eine Masse von 189 an. Da Kynurensäure dasselbe Molekulargewicht hat, das isolierte Produkt aber ein anderes chromatographisches Verhalten zeigt, nahmen wir an, daß es sich um eine stellungsisomere 5-oder 8-Hydroxy-chinaldinsäure handelt.

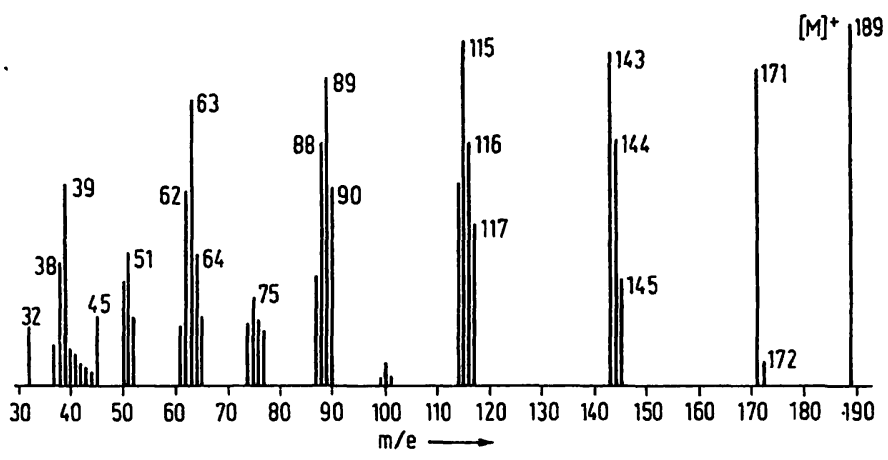

Abb. 4 Massenspektrum der isolierten $\begin{aligned} & \text { 8-Hydroxy-chinaldinsäure, aufge- } \\ & \text { nommen mit dem Varian MAT CH } 4\end{aligned}$

Durch Abspaltung von Wasser entsteht ein Massenpeak bei 171. Unter Berücksichtigung eines massenspektrometrischen orthoEffektes (10) kann das Proton der phenolischen Hydroxylgruppe in Stellung 8 des Chinolinringes mit der OH-Gruppe des Carboxyls in Stellung 2 als Wasser abgespalten werden. Erleichtert wird die Wasserabspaltung durch das benachbarte $\mathrm{N}$-Atom, das als Brücke zur Carboxylgruppe dient. Bei der 5-Hydroxy-chinaldinsäure ist eine analoge Wasserabspaltung nicht möglich. Die nur sehr schwache Intensität der Masse 172 erklärt sich durch die geringfügige Abspaltung von $\mathrm{OH}$ aus der Carboxylgruppe. Die Masse 144 entsteht durch Abspaltung der seitenständigen COGruppe. In geringem Umfang erfolgt auch aus dem Molekularpeak eine $\mathrm{CO}_{2}$-Abspaltung, wodurch die Masse 145 erklärt ist. $\mathrm{Da}$ im Molekül 2 acide Protonen angenommen werden müssen, sollte bei Behandlung mit $\mathrm{D}_{2} \mathrm{O}$ ein Molekülpeak erscheinen, der 2 Masseneinheiten größer ist. Dies konnte durch ein 2. Massenspektrum bewiesen werden: Der Molekülpeak verschiebt sich zur Masse 191. Budzikiewicz (11) fand bei 2-.4 und 8-Hydroxychinolinen primär eine intensive Abspaltung von $\mathrm{CO}$ aus dem Heterocyclus. Eine Bestätigung finden wir in den Massenzahlen 115, 116 und 117. Eine präformierte Chinolonform, wie z. B. bei der Masse 143, eliminiert leichter CO, daher ist die Intensität der Masse 115 größer als die von 116 und 117. Die Massen 88, 89 und 90 stellen charakteristische Bruchstücke der $\mathrm{N}$-haltigen Heterocyclen dar und entstehen durch Abspaltung von $\mathrm{C}_{2} \mathrm{H}_{2}, \mathrm{CO}$ und $\mathrm{CH}_{2} \mathrm{~N}$ aus der höheren Massengruppe um 115. Dem Bruchstück $\mathrm{C}_{7} \mathrm{H}_{5}$, Massenzahl 89, kommt nach Sprreller (12) die Struktur eines Dehydrotropylium-Ions zu. Ebenso chatakteristisch für diese Heterocyclen ist die Massengruppe um 63 (Massenzahl 62, $6^{3}$ und 64).
Das Massenspektrum bestätigt das Vorliegen einer Hydroxy-chinolin- bzw. iso-chinolin-carbonsäure. Unter Berücksichtigung des massenspektrometrischen orthoEffektes und des UV-Spektrums muß die Struktur einer 8-Hydroxy-chinolin-2-carbonsäure angenommen werden.

In der abgeleiteten Strukturformel befinden sich 5 Protonen am Chinolinkern. In einem NMR-Spektrum sollte sich für die beiden Protonen in Stellung 3 und 4 des Chinolinringes ein AB-System und für die 3 Protonen in Stellung 5, 6 und 7 ein ABC-System ausbilden.

Das Kernresonanzspektrum zeigt nun zwischen 8,62 und 8,11 ppm die 4 Linien eines AB-Systems an. Die Kopplungskonstante $\mathrm{J}_{3,4}=8,2 \mathrm{~Hz}$ stimmt gut mit Litcraturangaben (13) über orthoProtonen-Kopplung überein $(5-9 \mathrm{~Hz})$. Die Theorie ergibt, daß bei einer Iso-chinolin-Struktur $\mathrm{J}_{3,4}$ einen kleineren Wert haben $\mathrm{mu} B$, so $\mathrm{da} \beta$ eine Iso-chinolin-Struktur ausgeschlossen werden kann. Das ABC-System zeigt insgesamt 12 Banden an und besteht aus 3 Gruppen zu je 4 Banden gemäß den 3 benachbarten Protonen. Die Absorptionen liegen zwischen 7,74 und 7,18 ppm. Die 1. Gruppe zwischen 7,74 und 7,58 ppm ist dem mittleren Proton zuzuordnen, das in ortho-Kopplung tritt zu den beiden benachbarten Protonen. Die erhaltenen Kopplungskonstanten liegen auch hier im Bereich der Literaturwerte. Die außenstehenden Protonen (Stellung 5 und 7 des Chinolinringes) verursachen die 8 Banden zwischen 7,57 und 7,18 ppm. Da diese Protonen neben der ortho-Kopplung zu dem mittleren Proton auch eine metaKopplung gegenseitig haben, ist die beobachtete meta-Kopplungskonstante von $\mathrm{J}=2,0 \mathrm{~Hz}$ in guter Übereinstimmung mit Literaturangaben (13) $1-3 \mathrm{~Hz}$ ).

Das NMR-Spektrum läßt keine Aussage über die Position der Hydroxylgruppe zu. Diese ist sowohl in 5wie in 8-Stellung des Chinolinringes möglich.

Alle spektroskopischen Aussagen stehen in Übereinstimmung mit der Struktur einer 8-Hydroxy-chinolin2-carbonsäure.

Zum abschließenden Beweis wurde diese Substanz synthetisiert. Das aufgenommene IR- und Massenspektrum steht in Úbereinstimmung mit den Spektren des isolierten Produktes.

Die Synthese wurde zuerst nach der Methode von IRvING und Mitarbeitern (14) durchgeführt. Ein reineres Produkt und bessere Ausbeuten wurden nach der Synthese von Birringer (8) erhalten: Xanthurensäure wird mit $\mathrm{POCl}_{3}$ zur 4-Chlor-8-hydroxy-chinaldinsäure umgesetzt. Die isolierte $Z$ wischenstufe wird in $0,1 \mathrm{~N} \mathrm{KOH}$ gelöst und mit einem Pd/AktivkohleKatalysator mit Wasserstoff hydriert. $F_{p} 209^{\circ}$ (Zersetzung). Schmelzpunkt des isolierten Produktes aus dem Urin $207-208^{\circ}$. 


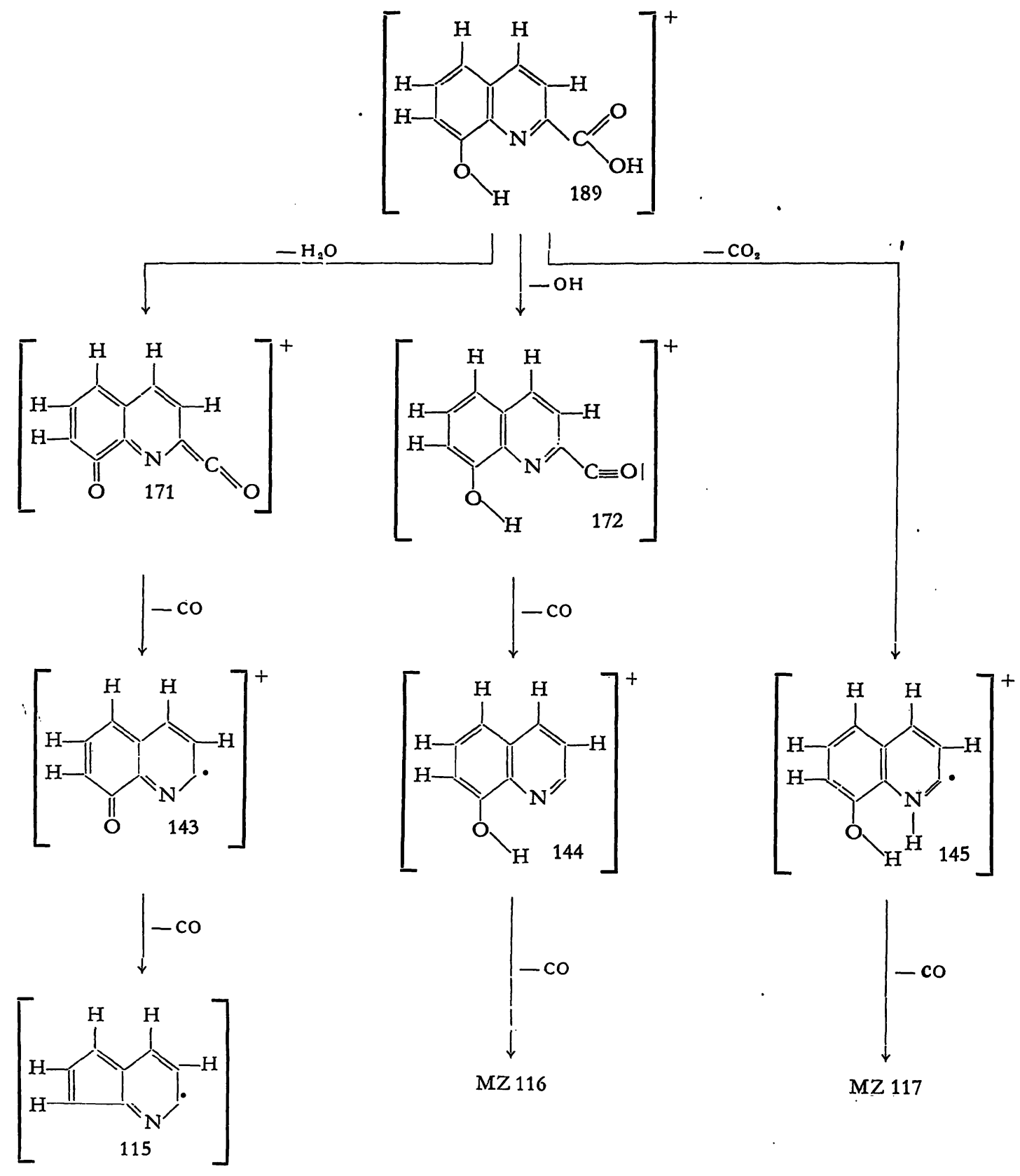

Abb. 5

Deutung der wichtigsten massenspektroskopischen Bruchstücke

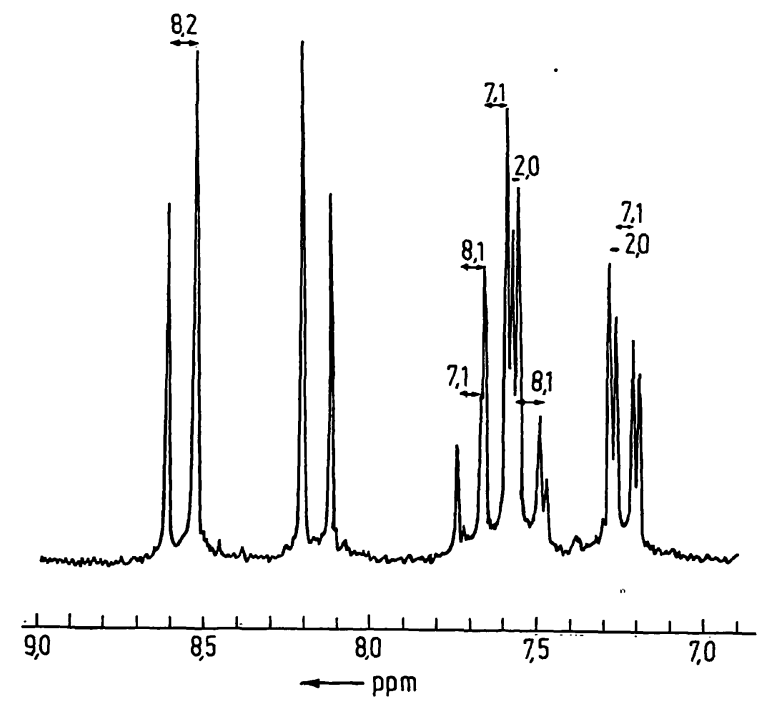

Abb. 6

NMR-Spektrum der isolierten 8-Hydroxy-chinaldinsäure. Lösungsmittel Hexadeutero-Dimethylsulfoxyd, aufgenommen mit dem Varian HA-100 
Untersuchungen bei den beiden lebenden Geschwisterkindern

Bei dem ältesten Geschwisterkind (2. Kind, 9 Jahre alt) ergeben die Tryptophan-Stoffwechseluntersuchungen eine Besonderheit. Im Basalurin werden die oxydativen Abbaumetabolite im Normbereich nachgewiesen, dagegen tritt nach Belastung keine vermehrte Ausscheidung von Kynurensäure, Xanthurensäure und 3Hydroxy-anthranilsäure ein. Die Ausscheidung von Tryptophan, Indolessigsäure und 5-Hydroxy-indolessigsäure ist sogar geringer als im Basalurin. Eine 2. Belastung 3 Wochen später bestätigt die Ergebnisse. Eine Deutung dieser Befunde ist z. Z. nicht möglich. Dieses Kind ist klinisch gesund, eine geistige und psychische Rückständigkeit muß jedoch festgestellt werden.

Die Tryptophan-Stoffwechseluntersuchungen bei dem 5. Kind ( 3 Monate alt) ergeben keine nennenswerten Besonderheiten. Prophylaktisch hatte das Kind seit seiner Geburt $10 \mathrm{mg}$ Pyridoxin/Tag erhalten. Die - Vitamin-B - -Gaben werden bei Klinikaufnahme abgesetzt. Die ab dem 5. Tag untersuchten Basalurine enthalten nur Kynurensäure $(0,8-1,0 \mathrm{mg} / 24 \mathrm{Stdn}$.), Xanthurensäure wird nur sporadisch ausgeschieden ( $\max .0,2 \mathrm{mg} / 24 \mathrm{Stdn}$.). Die Ausscheidung von überwiegend nur einem Metaboliten des oxydativen $\mathrm{Ab}-$ baus veranlaßte uns, 4 Tryptophan-Belastungen durchzuführen im zeitlichen Abstand von 6, 14 und 5 Tagen. Dabei zeigte sich, daß mit wiederholter Durchführung der Belastung die Ausscheidung der oxydativen Abbaumetabolite immer geringer wird, nur die Xanthurensäure-Ausscheidung nimmt zu. (10 Stdn. vor der 2. Belastung wurden einmalig $10 \mathrm{mg}$ Pyridoxin oral gegeben.) Zur Prüfung dieser Befunde werden bei einem 7 Monate alten Kontrollsäugling 2 Belastungen im

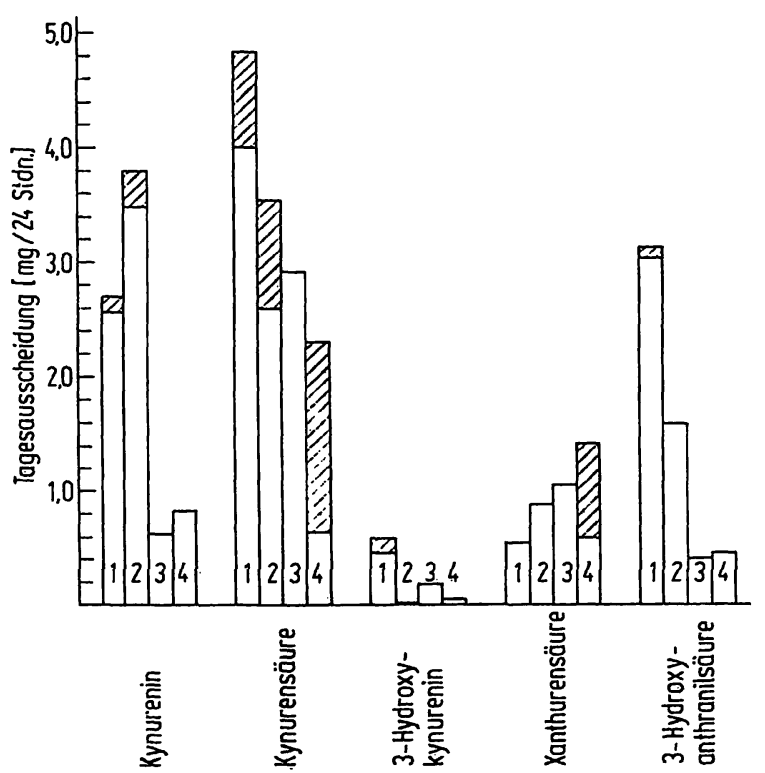

A.bb. 7

Fünftes Kind (klinisch gesund): Vergleich der Ausscheidung der Tryptophan-Metabolite nach 4 Belastungen. Bei der 1. und 2. Belastung wurde der 24-Stdn.-Sammelurin getrennt in zwei 12-Stdn.getrennt gesammelt. (Zehn Stdn. vor der 2. Belastung wurden $10 \mathrm{mg}$ Pyridoxin oral gegeben).
Abstand von 5 Tagen durchgeführt. Die vermehrt ausgeschiedenen Metabolite differieren bei beiden $\mathrm{Be}-$ lastungen um $\pm 10 \%$. Ob der Abnahme der Metaboliten-Ausscheidung bei dem 5 . Kind eine besondere Bedeutung zukommt, kann nicht gesagt werden.

\section{Diskussion}

Die Pathogenese der Vitamin- $\mathrm{B}_{6}$-abhängigen Krämpfe ist bis heute völlig ungeklärt. Untersuchungen haben keinen Anhalt für eine Störung des Pyridoxin-Stoffwechsels geliefert. Ein Zusammenhang zwischen diesen Krämpfen und dem Vitamin $B_{6}$ kann überzeugend nur aus der einen Tatsache abgeleitet werden, daß diese Krämpfe innerhalb von Minuten durch eine hohe Vitamin- $B_{6}$-Dosis kupiert werden können. Die Dauertherapie führt $z u$ einer normalen Entwicklung des Säuglings. Bei gleicher Symptomatik muß zwischen Vitamin- $\mathrm{B}_{6}$-abhängigen Krämpfen und Vitamin- $\mathrm{B}_{6}$ Mangelkrämpfen unterschieden werden.

Tab. 3

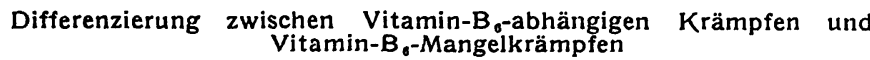

\begin{tabular}{|c|c|c|}
\hline & $\begin{array}{l}\text { Vitamin- } B_{\beta} \text {-abhän- } \\
\text { gige Krämpfe }\end{array}$ & $\begin{array}{c}\text { Vitamin- } \mathrm{B}_{\mathrm{B}}-\text { Mangel- } \\
\text { krämpfe }\end{array}$ \\
\hline Symptome & \multicolumn{2}{|c|}{$\begin{array}{l}\text { Hyperirritabilität - Hyperakusis - } \\
\text { Berührungsempfindlichkeit - generali- } \\
\text { sierte tonisch-klonische Krämpfe }\end{array}$} \\
\hline Krampfbeginn & $\begin{array}{l}\text { 1. bis 10. Lebenstag, } \\
\text { evtl. vor und unter } \\
\text { der Geburt }\end{array}$ & 1. bis 4. Monat \\
\hline $\begin{array}{l}\text { Erforderliche Vitamin- } \\
\text { B } \text {-Dosis }^{-}\end{array}$ & $\begin{array}{l}\text { hohe Dosen bis } \\
80 \mathrm{mg} / \mathrm{Tag}\end{array}$ & 0,2 bis $1,4 \mathrm{mg} / \mathrm{Tag}$ \\
\hline $\begin{array}{l}\text { Folgen einer verspäteten } \\
\text { Therapie }\end{array}$ & $\begin{array}{l}\text { schwere Cerebral- } \\
\text { schäden }\end{array}$ & $?$ \\
\hline $\begin{array}{l}\text { Recidiv nach Therapie- } \\
\text { abbruch }\end{array}$ & $\begin{array}{l}\text { nach 4-6 Tagen, } \\
\text { selten später }\end{array}$ & $\begin{array}{l}\text { nach } 6 \text { Monaten } \\
\text { und später }\end{array}$ \\
\hline Vererbung & autosomal-recessiv & nein \\
\hline Ursache & endogen & exogen und endogen \\
\hline Tryptophan-Belastung & $\begin{array}{l}\text { normale Xanthuren- } \\
\text { säure Ausscheidung }\end{array}$ & $\begin{array}{l}\text { erhöhte Xanthuren- } \\
\text { säure Ausscheidung }\end{array}$ \\
\hline
\end{tabular}

Abweichend von den meisten bisher berichteten Fällen traten die Krämpfe bei unserem Fall erst mit 31/2 Monaten auf. Die frühzeitig eingesetzte Vitamin- $\mathrm{B}_{6}-$ Dauertherapie führte $\mathrm{zu}$ einer normalen Entwicklung des Säuglings. Ein Auslaßversuch 3 Jahre später zeigte, daß diese Vitamin- $\mathrm{B}_{6}$-Abhängigkeit immer noch bestand: 12 Tage nach Therapieabbruch Grand-Mal Anfall. Die Untersuchung des Tryptophan-Stoffwechsels führt zu folgenden Ergebnissen:

Unter der Vitamin- $B_{6}$-Dauertherapie ist eine im wesentlichen normale Ausscheidung der Metabolite festzustellen. Die Unterbrechung der täglichen PyridoxinZufuhr führt zu einer verminderten Ausscheidung der Metabolite des oxydativen Abbaus.

Die hohe Pyridoxin-Dosis, die zur Kupierung des nach 12 Tagen auftretenden Grand-Mal-Anfalles injiziert wird, führt nicht wieder zu dem erwarteten normalen Ausscheidungsmuster wie es unter der Vitamin- $\mathrm{B}_{6}$ Dauertherapie erhalten wird. Sogar neun Tage nach dem Anfall werden keine Metabolite des oxydativen Abbaus ausgeschieden, obwohl die Dauertherapie wieder durchgeführt wird. Das Kind ist klinisch völlig 
unauffällig, dennoch muß eine biochemische Störung weiter bestehen. Erst eine Urinuntersuchung am 21. Tag nach dem Anfall ergibt ein Ausscheidungsmuster, das identisch ist mit der 1. Untersuchung (Abb. 1, A) unter Dauertherapie. Die Normalisierung muß also $z$ wischen dem 10. und 20. Tag eingetreten sein.

Abgesehen vom Tryptophan-Stoffwechsel ergibt die dünnschichtchromatographische Untersuchung der Urine vor, während und nach dem Krampfanfall keine nennenswerten Unterschiede (Abb. 1, C, D und E). Nur in dem Urin am Tage des Anfalles werden in der 1. Fraktion der säulenchromatographischen Urinauttrennung zahlreiche, z. T. instabile Substanzen festgestellt, die in den Urinen $C$ und $E$ nicht nachzuweisen sind. Infolge der zur Verfügung stehenden geringen Mengen konnten diese Substanzen nicht näher untersucht werden. Wird diese 1. Fraktion nach 3 tägiger Gefriertrocknung und Auflösung in physiologischer $\mathrm{NaCl}$-Lösung in eine Maus injizic rt, so ist eine auffällige motorische Unruhe des Tieres über $40 \mathrm{Min}$. zu beobachten. Ein Parallelversuch mit der 1. Fraktion aus Urin $\mathrm{E}$ verlauft ohne Wirkung auf das Verhalten des Tieres.

Die erst nach Absetzen der Vitamin- $\mathrm{B}_{6}$-Therapie ausgeschiedene und kurz vor dem Krampfanfall vermehrt im Urin nachzuweisende 8-Hydroxy-chinaldinsäure berechtigt zu der Vermutung, daß ein noch unbekannter Zusammenhang zwischen der Biogenese dieses Metaboliten und den Vitamin- $\mathrm{B}_{6}$-abhängigen Krämpfen besteht.

Nach der chemischen Struktur könnte die 8-Hydroxychinaldinsäure aus dem Metabolismus des Tryptophan abgeleitet werden, $z$. B. durch Dehydroxylierung in Stellung 4 der Xanthurensäure. Aber auch die Möglichkeit einer Biosynthese aus 3-Hydroxy-anthranilsäure muß diskutiert werden.

Rothstein und Greenberg (15) fanden 1957, daß Xanthurensäure-[4-14 C] bei der Ratte zu $100 \%$ als unverändertes Produkt und als Mono- und Diglucuronid in Konjugation mit Serin ausgeschieden wird. Zusätzliche Vitamin- $\mathrm{B}_{6}-\mathrm{Gabe}_{\mathrm{i}}$ haben auf das Ergebnis keinen Einfluß. Dagegen stellte Takahashr (16) 1958 eine geringe Umwandlung in der Ratte zu 8-Hydroxy-
chinaldinsäure-[4-14C] fest $(2-4 \%)$. Der Nachweis dieses Metaboliten im menschlichen Urin nach Xanthurensäure-Belastung gelang nicht überzeugend, spektroskopische Angaben fehlen. 1962 wies KarHARA (17) nach, daß markierte Xanthurensäure in Kaninchen $\mathrm{zu} 70-80 \%$ bei oraler und nur zu $3-10 \%$ bei subkutaner Injektion in die 8-Hydroxy-chinaldinsäure umgewandelt wird. Die Dehydroxylierung soll sogar weiter bis zur Chinaldinsäure verlaufen $(1-2 \%)$. Dementsprechend stellte er bei der Verabreichung von Kynurensäure eine hohe Umwandlung zu Chinaldinsäure fest. Beim Menschen und bei der Ratte sollen $29 \%$ einer oralen Dosis Kynurensäure als Chinaldinsäure ausgeschieden werden (18). Nach intraperitonealer Injektion von markierter Kynurensäure fand MurachI (19) bei Tierversuchen nur eine Umwandlungsrate von $\max .5 \%$. Ein neues Konjugat beschrieben KaIhara und PrICE (20): 25\% einer oralen Dosis Kynurensäure werden bei der Katze als Chinaldylglycyltaurin ausgeschieden, dagegen nur $1 \%$ bei subcutaner Injektion. Eine Hydroxylierung der Chinaldinsäure zu Kynuren- oder Xanthurensäure wurde bisher nicht beobachtet $(21,22)$.

Es kann als gesichert gelten, daß Chinaldin-, Kynurenund Xanthurensäure im tierischen Organismus keinem Abbau des aromatischen Systems unterliegen. Demgegenüber steht die Fähigkeit von Bakterien, Kynurensäure über 7,8-Dihydroxykynurensäure in Glutaminsäure, Alanin, Essigsäure und $\mathrm{CO}_{2}$ abzubauen (23). Der Mechanismus, über den die Dehydroxylierung der Kynuren- und Xanthurensäure verläuft, ist völlig unklar. Die Einwirkung von Darmbakterien ist nicht auszuschließen $(24,25)$.

In diesem Zusammenhang mögen die Dehydroxylierungen von Brenzcatechin-Verbindungen erwähnt werden: 3.4-Dihydroxybenzoeșäure (26), 3.4-Dihydroxy-phenylessigsäure (27) und die 3.4-Dihydroxyzimtsäure (28) werden in geringer Menge in die metabzw. para-Hydroxyverbindung umgewandelt. Nach partieller Reduzierung des aromatischen Ringes ist hier als Zwischenstufe ein trans-Diol anzunehmen, aus dem sich durch Wasserabspaltung die mono-Hydroxyverbindung bildet.<smiles>NC(Cc1c[nH]c2ccccc12)C(=O)O</smiles><smiles>NC(C(=O)O)C(=O)O</smiles><smiles>O=C(O)c1cc(O)c2cccc(O)c2n1</smiles><smiles>CC(C)(C)OC(C)(C)C</smiles>

Z. klin. Chem. u. klin. Bịochem. / 9. Jahrg. 1971 / /Heft 2 
Unter der Annahme, daß im Verdauungstrakt durch Darmbakterien die Dehydroxylierung der Xanthurensäure zu 8-Hydroxy-chinaldinsäure erfolgt, erhebt sich die Frage, warum diese Umwandlung bei dem von uns untersuchten Fall mit Vitamin- $\mathrm{B}_{6}$-abhängigen Krämpfen erst nach Absetzen der Vitamin- $\mathrm{B}_{6}$-Therapie einsetzt. Ein Zusammenhang $z$ wischen Vitamin $B_{6}$ und der Tätigkeit der Bakterien ist nicht bekannt. In den letzten vier $24 \mathrm{Stdn}$.-Urinen vor dem Krampfanfall sind etwa $15 \mathrm{mg}$ 8-Hydroxy-chinaldinsäure ausgeschieden worden, dagegen nur sehr geringe Mengen von Xanthurensäure. Bisher ist es uns nicht gelungen, diesen Metaboliten im Urin von Kindern und Erwachsenen ein- deutig nachzuweisen. Auch Hydrolyse des Urins führte zu keinem positiven Nachweis.

Die Ableitung der 8-Hydroxy-chinaldinsäure aus dem Tryptophan-Stoffwechsel müßte u. E. bei unserem beschriebenen Fall durch einen Versuch mit markierten Tryptophan überzeugend bewiesen werden. Aus humanitären Gründen können aber die Versuchsbedingungen bei dem Kind nicht wiederholt werden.

Mit Unterstützung der Deutschen Forschungsgemeinschaft. Herrn Dr. SErdr (BASF, Ludwigshafen) danke ich für die Durchführung der massenspektroskopischen Untersuchungen und des NMR-Spcktrums.

\section{Literatur}

1. Hinkel, G. K., H. W. KintzeL und A. KNAPp, Schweiz. med. Wschr. 100, 1152 (1970). - 2. Scriver, $C_{H}$. R., Pediatrics, Springfield, 26, 62 (1960). - 3. Gentz, J., A. Hampelt, S. Johansson, S. Lindstedt und B. Person, Acta Paediatrica Scand 56, 17 (1967). - 4. KRUSE, R., W. Kochen und D. FeIst, Neuropaediatrie i. Druck. - 5. Kochen, W. und K. Hochberg, Zschr. Krebsforsch. 73, 25 (1970). - 6. KocrEEN, W., in Vorbereitung. 7. Scotr, I. A., Interpretation of the Ultraviolett Spectra of Natural Products. In: Barton, D. H. R. und Doering, W., Intern. Series of Monographs of Organic Chemistry, Vol. 7, Pergamon Press, Oxford-London-Edinburgh-New York-Paris-Frankfurt (1964). - 8. Birringer, H., Diss. Heidelberg 1969 (Untersuchungen über die Wehrchemie des Schwimmkäfers Ilybius Fenestratus). - 9. NAKANISHr, K., Infrared Absorption Spectroscopy, Holden Day, Inc., San Francisco und Nankodo Comp. LTD, Tokyo (1962). - 10. EMERY, E. M., Analytic. Chem. 32, 1495 (1960). - 11. Budzikiewicz, H., C. DJerassi und D. H. Williams, Mass Spectrometry of Organic Compounds, Holden Day, Inc., San Francisco-London-Cambridge-Amsterdam (1967). 12. SpIteller, G. Massenspektrometrische Strukturanalyse organischer Verbindungen, Verlag Chemie, Weinheim/Bergstr. (1966). - 13. SUHR, H., Anwendung der kernmagnetischen
Resonanz in der organischen Chemie, Springer Verlag, Berlin (1965). - 14. Ir.jing, H. und A. R. Pinnington, J. Chem. Soc. 3782 (1954) - 15. Rothstein, M. und D. M. Greenserg, Arch. Biochen. Biophysics 68, 206 (1957). - 16. TakaHashi, H. und J. M. PrICE, J. biol. Chemistry 233, 150 (1958). - 17. KaIhara, M. und J. M. Price, J. biol. Chemistry 237, 1727 (1962). 18. Takahasian, H., M. Kaifiara und J. M. Price, J. biol. Chemistry 223, 705 (1956). - 19. Murachi, T., K. Tsukada und O. Hayaishi, Biochemistry, USA, 2, 304 (1963). - 20. Kaimara, M. und J. M. PrICE, J. biol. Chemistry 236, 508 (1961). - 21. Ellinger, A. und Z. Matsuoka, Hoppe-Seyler Z. physiol. Chem. 109, 259 (1920). - 22. Kaimara, M., J. biol. Chemistry, 235, 136 (1960). - 23. Taniucrir, H. und O. Hayaishi, J. biol. Chemistry, 238, 283 (1963). - 24. Booth, A. N., Proc. Soc. exp. Biol. Med. 120 (2), 546 (1965). - 25. KarriarA, M. und J. M. Price, J. biol. Chemistry. 238, 4082 (1963). - 26. DeEds, F., A. N. Bootri und F. T. Jones, J. biol. Chemistry, 225, 615 (1957). 27. Booth, A. N., C. W. Murray, F. DeEds und F. T. Jones, Federation Proc. 14, 321 (1955). - 28. Booth, A. N., O. H. Emerson, F. T. Jones und F. DeEds, J. biol. Chemistry, 229, 51 (1957).
Dr. W. Kochen Univ. Kinderklinik, Isotopenabt. 69 Heidelberg, Hofmeisterweg 